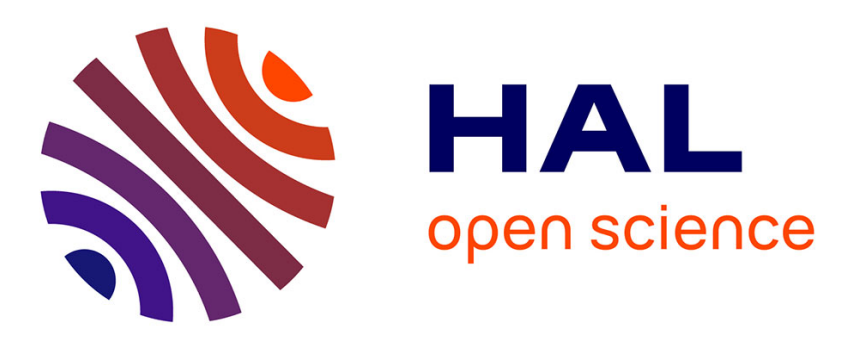

\title{
Balanced realization of lossless systems: Schur parameters, canonical forms and applications
}

\author{
Ralf L.M. Peeters, Martine Olivi, Bernard Hanzon
}

\section{To cite this version:}

Ralf L.M. Peeters, Martine Olivi, Bernard Hanzon. Balanced realization of lossless systems: Schur parameters, canonical forms and applications. IFAC Symposium on System Identification, SYSID 2009, Jul 2009, Saint-Malo, France. pp.273-283, 10.3182/20090706-3-FR-2004.00045 . hal-00753676

\section{HAL Id: hal-00753676 \\ https://hal.inria.fr/hal-00753676}

Submitted on 19 Nov 2012

HAL is a multi-disciplinary open access archive for the deposit and dissemination of scientific research documents, whether they are published or not. The documents may come from teaching and research institutions in France or abroad, or from public or private research centers.
L'archive ouverte pluridisciplinaire HAL, est destinée au dépôt et à la diffusion de documents scientifiques de niveau recherche, publiés ou non, émanant des établissements d'enseignement et de recherche français ou étrangers, des laboratoires publics ou privés. 


\title{
Balanced realization of lossless systems: Schur parameters, canonical forms and applications
}

\author{
Ralf Peeters * Martine Olivi ${ }^{* *}$ Bernard Hanzon *** \\ * Department of Knowledge Engineering, Maastricht University, \\ P.O. Box 616, 6200 MD Maastricht, The Netherlands, \\ ralf.peeters@maastrichtuniversity.nl \\ ** INRIA, Projet APICS, BP 93, 06902 Sophia-Antipolis Cedex, \\ France \\ *** School of Mathematical Sciences, University College Cork, Cork, \\ Ireland
}

\begin{abstract}
Lossless systems have many applications in systems and control theory, including signal processing, filter design, system identification, system approximation, and the parameterization of classes of linear systems. In this survey paper we address the issue of parameterization of the space of rational lossless matrix functions by successfully combining two approaches. The first approach proceeds in state-space from balanced realizations and triangular pivot structures of reachability matrices. The second approach concerns interpolation theory with linear fractional transformations and the tangential Schur algorithm. We construct balanced realizations (and canonical forms) in terms of the Schur parameters encountered in the tangential Schur algorithm, and conversely, we interpret balanced realizations in discrete-time and in continuous-time in terms of Schur parameters. A number of application areas are discussed to illustrate the importance of this theory for a variety of topics.
\end{abstract}

Keywords: Lossless systems, balanced realization, interpolation theory, Schur parameters, canonical forms.

\section{INTRODUCTION}

Lossless systems of finite order have applications in various areas of linear systems and control theory. In the area of system identification, approximation and model reduction, they have for instance been used in connection with the Douglas-Shapiro-Shields factorization, see e.g., Douglas et al. (1970); Baratchart (1990); Leblond and Olivi (1998); Fulcheri and Olivi (1998), to perform $\mathrm{H}_{2}$-model order reduction of stable linear systems. They feature in the separable least squares method for system identification, see Bruls et al. (1999), where the identification problem is reduced to an optimization problem over the set of input normal pairs. In the design of orthogonal wavelets (and multiwavelets) from filter banks, see Strang and Nguyen (1996), they play a central role as the lossless polyphase matrices, used to filter the phases of an input signal to produce a decompostion into approximation and detail coefficients. See Vaidyanathan and Doğanata (1989) for a tutorial on applications of lossless systems in digital signal processing.

Lossless systems also play an important role in the construction of parameterizations of various classes of linear systems, with special properties of these classes, such as stability, built in. See, e.g., Ober (1987b); Hanzon and Ober (1998). In this context, balanced realizations of lossless systems have been pursued. Balanced realizations are well known to have numerical advantages and to be useful for model reduction, especially in conjunction with balance-and-truncate type procedures.

A balanced canonical form for SISO lossless systems in continuous-time was presented in Ober (1987a); see also Ober (1987b). In the constructions of Hanzon and Ober (1997, 1998) for other classes of systems, the case of continuous-time lossless systems also plays a central role. In the SISO case, the resulting canonical form for lossless systems is balanced with a positive upper triangular reachability matrix. In the MIMO case, Kronecker indices and nice selections are used to arrive at balanced overlapping canonical forms.

For discrete-time lossless systems, canonical forms can be obtained indirectly by transfering results from the continuous-time domain to the discrete-time domain by application of a bilinear transformation. However, this approach destroys certain nice properties of the continuoustime canonical form: truncation of state components no longer leads to reduced order systems that are balanced and in canonical form, and upper triangularity of the reachability matrix is lost. In Hanzon and Peeters (2000), similar ideas are applied to the SISO discrete-time lossless case directly. This produces a balanced canonical form with the desired properties, which can in fact be parametrized using Schur parameters.

The class of lossless transfer functions of finite order is bijectively related to the class of rational inner functions, 
of which the differential structure is studied in Alpay et al. (1994). There, a parametrization for the MIMO case is obtained by means of a recursive procedure, the tangential Schur algorithm, which involves Schur parameter vectors, interpolation points and normalized direction vectors.

In Hanzon et al. (2006) this interpolation theoretic approach was successfully combined with the balanced statespace approach, to deal with the MIMO discrete-time lossless case directly. In that paper, the atlases developed in Alpay et al. (1994) are supplied with balanced statespace realizations in terms of the parameters used in the tangential Schur algorithm. It involves linear fractional transformations which replace the unitary matrix multiplications introduced in Hanzon and Peeters (2000).

In Peeters et al. (2007) it was investigated which choices for the parameters in the tangential Schur algorithm enable one to deal with specified Kronecker structures, thereby generalizing positive upper triangularity of the reachability matrices to canonical forms with pivot structures.

The interpretation of continuous-time balanced realizations of lossless functions exhibiting an upper triangular reachbility matrix in terms of interpolation theory and Schur parameters, turns out to involve interpolation conditions at the stability boundary, more specifically at infinity. In Hanzon et al. (2008) the original continuous-time canonical form of Ober (1987a) was analyzed in detail in this way, using the results of Ball et al. (1990) on boundary interpolation problems. A generalization of this approach to the MIMO case completes the picture and is currently under preparation.

In this paper we present a survey of the above results in a unified way. In Section 2 we present a background on realization theory and balanced realizations of stable systems. Section 3 deals with lossless function as well as with $J$-inner functions, which also play an important role in the interpolation theoretic approach to the parameterization of lossless systems. In Section 4 the tangential Schur algorithm is developed, and the various results connecting balanced realizations and Schur parameters are presented. Section 5 provides details on application areas and Section 6 concludes the paper.

\section{BALANCED STATE-SPACE REALIZATIONS OF LINEAR SYSTEMS}

\subsection{Realization theory and Lyapunov balancing in discrete time}

Consider a linear time-invariant state-space system in discrete time, with $m$ inputs and $p$ outputs:

$$
\begin{aligned}
& x_{t+1}=A x_{t}+B u_{t}, \\
& y_{t}=C x_{t}+D u_{t} .
\end{aligned}
$$

Here we have: $t \in \mathbb{Z}$ (discrete time); $x_{t} \in \mathbb{C}^{n}$ (the state at time $t$ ) for some nonnegative integer $n$ (the state space dimension); $u_{t} \in \mathbb{C}^{m}$ (the input at time $t$ ) and $y_{t} \in \mathbb{C}^{p}$ (the output at time $t$ ). The sizes of the complex-valued matrices $A, B, C$ and $D$ are $n \times n, n \times m, p \times n$ and $p \times m$, respectively. The $p \times m$ rational transfer matrix of such a system is given by $G(z)=D+C\left(z I_{n}-A\right)^{-1} B$.
Two state-space systems are called input-output equivalent if they have the same transfer matrix. Here, two rational (matrix) functions are identified if they agree almost everywhere, i.e.: common factors are always canceled. Note that a transfer matrix in this causal set-up is always proper (i.e., analytic at infinity), the direct feedthrough (gain) $D$ being the value at infinity.

Realization theory establishes that, conversely, any $p \times m$ rational matrix function $G(z)$ analytic at infinity can be written in the form

$$
G(z)=D+C\left(z I_{n}-A\right)^{-1} B
$$

for some quadruple of matrices $(A, B, C, D)$, then called a state-space realization of $G(z)$. To such a realization we associate the block-partitioned realization matrix $R$ :

$$
R=\left(\begin{array}{ll}
D & C \\
B & A
\end{array}\right)
$$

A realization $(A, B, C, D)$ of $G(z)$ is called minimal if no other realization of $G(z)$ exists for which the statespace dimension $n$ is smaller. This minimal state-space dimension $n$ is then called the order of the system; it is equal to the McMillan degree of the transfer function. Two minimal realizations $(A, B, C, D)$ and $\left(A^{\prime}, B^{\prime}, C^{\prime}, D^{\prime}\right)$ of a given function $G(z)$ are always similar: there exists a unique invertible matrix $T$ such that $\left(A^{\prime}, B^{\prime}, C^{\prime}, D^{\prime}\right)=$ $\left(T A T^{-1}, T B, C T^{-1}, D\right)$; equivalently:

$$
\left(\begin{array}{ll}
D^{\prime} & C^{\prime} \\
B^{\prime} & A^{\prime}
\end{array}\right)=\left(\begin{array}{cc}
I_{p} & 0 \\
0 & T
\end{array}\right)\left(\begin{array}{ll}
D & C \\
B & A
\end{array}\right)\left(\begin{array}{cc}
I_{m} & 0 \\
0 & T^{-1}
\end{array}\right) .
$$

As is well-known, an input pair $(A, B)$ is reachable if the associated $n \times n m$ reachability matrix $K=$ ( $\left.B A B \ldots A^{n-1} B\right)$ has full row rank $n$. An output pair $(C, A)$ is observable if the $p n \times n$ observability matrix $O=\left(\begin{array}{c}C \\ C A \\ \vdots \\ C A^{n-1}\end{array}\right)$ has full column rank $n$. A state-space realization $(A, B, C, D)$ is minimal if and only if $(A, B)$ is reachable and $(C, A)$ is observable; cf., e.g., Kailath (1980). In this case, the poles of $G(z)$ are the eigenvalues of $A$, and its McMillan degree is equal to the sum of the degrees (multiplicities) of all the poles of $G(z)$ (see (Kailath, 1980, Sect. 6.5)). This definition for the McMillan degree generalizes to the larger class of proper and non-proper rational functions by including a possible pole at infinity with its associated degree.

Let $(A, B, C, D)$ be some realization of a transfer function. If the eigenvalues of $A$ all belong to the open unit disk, then the matrix $A$ is called (discrete-time) asymptotically stable, and $(A, B, C, D)$ an asymptotically stable realization. If $(A, B, C, D)$ is an asymptotically stable realization, then the controllability Gramian $W_{c}$ and the observability Gramian $W_{o}$ are well defined as the exponentially convergent series

$$
\begin{aligned}
& W_{c}=\sum_{k=0}^{\infty} A^{k} B B^{*}\left(A^{*}\right)^{k}, \\
& W_{o}=\sum_{k=0}^{\infty}\left(A^{*}\right)^{k} C^{*} C A^{k} .
\end{aligned}
$$


These Gramians are the unique (and positive semidefinite) solutions of the respective Lyapunov-Stein equations

$$
\begin{aligned}
& W_{c}-A W_{c} A^{*}=B B^{*}, \\
& W_{o}-A^{*} W_{o} A=C^{*} C .
\end{aligned}
$$

Moreover, under asymptotic stability of $A$ it holds that $W_{c}$ is positive definite if and only if the pair $(A, B)$ is reachable, and $W_{o}$ is positive definite if and only if the pair $(C, A)$ is observable. A minimal and asymptotically stable realization $(A, B, C, D)$ of a transfer function is called a balanced realization if its observability and controllability Gramians are both diagonal and equal. Then $W_{c}=W_{o}=$ $\operatorname{diag}\left\{\sigma_{1}, \sigma_{2}, \ldots, \sigma_{n}\right\}$ in which the positive real quantities $\sigma_{1}, \sigma_{2}, \ldots, \sigma_{n}$ denote the (Hankel) singular values of the system.

This concept of (Lyapunov) balanced realizations was first introduced in Moore (1981) in the continuous-time case and used for model reduction. In Pernebo and Silverman (1982) the same was done for the discrete-time case. The concept of balancing has since been extended to several other classes of linear systems as well as to nonlinear systems. Balanced realizations are now a well-established tool which often exhibit good numerical properties.

Any realization $(A, B, C, D)$ which is minimal and asymptotically stable, is known to be similar to a balanced realization. Such a balanced realization is in general not unique. Similarity transformation matrices $T$ which do not affect balancing are permutation matrices (allowing one to order the squared Hankel singular values on the main diagonal of $W_{c}=W_{o}$ ) and diagonal scaling matrices with unimodular entries along the main diagonal (including sign matrices). If some singular value has a multiplicity larger than one, other transformation matrices exist which do not affect balancing too.

\subsection{The continuous-time case and the bilinear transform}

A continuous-time state-space system $(A, B, C, D)$ refers to the set of equations

$$
\begin{aligned}
& \dot{x}_{t}=A x_{t}+B u_{t}, \\
& y_{t}=C x_{t}+D u_{t},
\end{aligned}
$$

with $t \in \mathbb{R}$. Here, the transfer matrix is similarly given by $G(s)=D+C\left(s I_{n}-A\right)^{-1} B$ and the concepts of minimality, reachability, observability and input-output equivalence are introduced entirely analogous to the discrete-time case.

A continuous-time system $(A, B, C, D)$ and the matrix $A$ are called asymptotically stable if the eigenvalues of $A$ are all located in the open left half of the complex plane. If $(A, B, C, D)$ is an asymptotically stable realization, then the controllability Gramian $W_{c}$ and the observability Gramian $W_{o}$ are well defined as the exponentially convergent integrals

$$
\begin{aligned}
& W_{c}=\int_{-\infty}^{\infty} e^{A t} B B^{*} e^{A^{*} t} d t, \\
& W_{o}=\int_{-\infty}^{\infty} e^{A^{*} t} C^{*} C e^{A t} d t .
\end{aligned}
$$

These Gramians are the unique (and positive semidefinite) solutions of the Lyapunov equations

$$
\begin{aligned}
& A W_{c}+W_{c} A^{*}=-B B^{*}, \\
& A^{*} W_{o}+W_{o} A=-C^{*} C .
\end{aligned}
$$

Under continuous-time asymptotic stability of $A$ it holds too that $W_{c}$ is positive definite if and only if the pair $(A, B)$ is reachable, and $W_{o}$ is positive definite if and only if the pair $(C, A)$ is observable. Once again, a minimal and asymptotically stable realization $(A, B, C, D)$ of a transfer function $G(s)$ is called a balanced realization if its observability and controllability Gramians are both diagonal and equal.

A well-known way to transfer results from discrete-time to continuous-time and vice versa, is by means of the bilinear transform $m: \mathbb{C} \rightarrow \mathbb{C}$, given by:

$$
m: z \mapsto s=\frac{z-1}{z+1},
$$

of which the inverse is given by $m^{-1}: s \mapsto z=\frac{1+s}{1-s}$. If $(A, B, C, D)$ is a discrete-time stable realization of a transfer matrix $G(z)$, then the transfer matrix $\widehat{G}(s)=$ $G\left(\frac{z-1}{z+1}\right)$ has a continuous-time stable state-space realization $(\widehat{A}, \widehat{B}, \widehat{C}, \widehat{D})$ given by

$$
\left(\begin{array}{ll}
\widehat{D} & \widehat{C} \\
\widehat{B} & \widehat{A}
\end{array}\right)=\left(\begin{array}{cc}
D-C\left(A+I_{n}\right)^{-1} B & \sqrt{2} C\left(A+I_{n}\right)^{-1} \\
\sqrt{2}\left(A+I_{n}\right)^{-1} B & \left(A-I_{n}\right)\left(A+I_{n}\right)^{-1}
\end{array}\right)
$$

Conversely it holds that:

$$
\left(\begin{array}{ll}
D & C \\
B & A
\end{array}\right)=\left(\begin{array}{cc}
\widehat{D}+\widehat{C}\left(I_{n}-\widehat{A}\right)^{-1} \widehat{B} & \sqrt{2} \widehat{C}\left(I_{n}-\widehat{A}\right)^{-1} \\
\sqrt{2}\left(I_{n}-\widehat{A}\right)^{-1} \widehat{B} & \left(I_{n}+\widehat{A}\right)\left(I_{n}-\widehat{A}\right)^{-1}
\end{array}\right)
$$

In fact, if $W_{c}$ and $W_{o}$ denote the Gramians for the discrete-time system $(A, B, C, D)$, then they also constitute the Gramians for the associated continuous-time system $(\widehat{A}, \widehat{B}, \widehat{C}, \widehat{D})$, showing that $(A, B, C, D)$ is discretetime balanced if and only if $(\widehat{A}, \widehat{B}, \widehat{C}, \widehat{D})$ is continuous-time balanced.

\subsection{Model order reduction: balance-and-truncate}

A popular and relatively easy technique for model order reduction within the class of stable linear systems, is offered by the balance-and-truncate approach. First a balanced realization $(A, B, C, D)$ is computed for which the Gramians are monotonically decreasing along their main diagonal. Then the original model order $n$ is reduced to a desired lower order $k<n$ by mere truncation of the state vector, retaining only its first $k$ entries. The matrices $A, B$ and $C$ are likewise replaced by their corresponding left upper submatrices $A_{11}, B_{1}$ and $C_{1}$, of sizes $k \times k, k \times m$ and $p \times k$, respectively. The popularity of this method is largely due to the computational simplicity of the method and the possibility to choose a reduced model order by monitoring and thresholding the values on the main diagonal of the Gramians, in combination with the experience that the approximations are often quite good in practice. It is shown in Glover (1984) that an $L_{\infty}$-error bound is available for the approximations. A drawback of the approach is the lack of an explicit optimization criterion which is minimized by this procedure. When a preferred 
approximation criterion is available, approximations of the balance-and-truncate type can also be used as a starting point for a numerical local search technique.

Both in discrete-time and in continuous-time, the balanceand-truncate procedure is easily seen to yield a stable approximation of order $\leq k$. It may happen, though, due to loss of reachability or observability that the reduced model order is actually less than $k$. For certain subclasses of systems, however, it can be guaranteed that the reduced model order does in fact equal $k$ by putting additional constraints on the parameterized form of the realization $(A, B, C, D)$. We shall encounter such a situation for some parameterizations of the class of lossless systems, discussed below. Finally, note that model order reduction by the balance-and-truncate procedure in general does not commute with the bilinear transformation between discrete-time and continuous-time.

\section{LOSSLESS SYSTEMS AND $J$-INNER FUNCTIONS}

3.1 Discrete-time lossless, inner, J-lossless and J-inner functions

When working in discrete-time, for any rational matrix function $R(z)$ we shall define the associated matrix functions $R^{*}(z)$ and $R^{\sharp}(z)$ by

$$
R^{*}(z):=R(\bar{z})^{*}, \quad \text { and } \quad R^{\sharp}(z):=R^{*}\left(z^{-1}\right) .
$$

Discrete-time lossless matrix functions are introduced according to the following definition.

Definition 3.1. In discrete-time, a square $p \times p$ rational matrix function $G(z)$ is called lossless (or stable all-pass) if at all points of analyticity it holds:

$$
\begin{aligned}
& G(z)^{*} G(z) \geq I_{p}, \quad \text { for }|z|<1, \\
& G(z)^{*} G(z)=I_{p}, \quad \text { for }|z|=1, \\
& G(z)^{*} G(z) \leq I_{p}, \quad \text { for }|z|>1 .
\end{aligned}
$$

Of these three conditions, each pair actually implies the remaining third condition and the inequalities are all to be understood in the sense of the partial ordering of positive semi-definite Hermitian matrices. Note that the Bode amplitude (gain) diagram for a SISO lossless system is constant 1 , for all $z=e^{i \omega}$, from which this class of systems derives its name.

The poles of a rational lossless function are all located inside the open unit disk. A lossless function is invertible: it holds that $G(z)^{-1}=G^{\sharp}(z)$. When $G(z)$ is lossless, also $G^{*}(z)$ and $G(z)^{*}$ are lossless. Note that a lossless function $G(z)$ is unitary at each point $z$ on the unit circle. The determinant of a lossless matrix is a scalar lossless function of the same McMillan degree. As we shall see below, a lossless matrix of McMillan degree $n$ can always be factored into a product of $n$ lossless matrices of McMillan degree 1, its so-called Potapov factorization.

Lossless functions have many applications in systems and control theory, including system identification and digital signal processing. See Vaidyanathan and Doğanata (1989) for a tutorial and many further properties of lossless systems. See also Genin et al. (1983).
When dealing with lossless (matrix) functions, we shall make use of interpolation theory. To this end it shall be convenient to generalize the concept of lossless functions in the following way, see also Potapov (1960); Dym (1989); Genin et al. (1983). Let $\Sigma$ be a $k \times k$ signature matrix, i.e., a diagonal matrix having only the entries \pm 1 along its main diagonal.

Definition 3.2. Let $\Sigma$ be a $k \times k$ signature matrix. Then a $k \times k$ rational matrix function $\Theta(z)$ is called discrete-time $\Sigma$-lossless if at all points of analyticity it holds:

$$
\begin{array}{ll}
\Theta(z)^{*} \Sigma \Theta(z) \geq \Sigma, & \text { for }|z|<1 \\
\Theta(z)^{*} \Sigma \Theta(z)=\Sigma, & \text { for }|z|=1 \\
\Theta(z)^{*} \Sigma \Theta(z) \leq \Sigma, & \text { for }|z|>1
\end{array}
$$

As before, this definition contains redundancy in the sense that each one of these three defining properties is implied by the other two.

Any rational matrix function $\Theta(z)$ which satisfies the property $\Theta(z)^{*} \Sigma \Theta(z)=\Sigma$ for $|z|=1$ is called $\Sigma$-unitary. For such functions the inverse is given by $\Theta(z)^{-1}=$ $\Sigma \Theta^{\sharp}(z) \Sigma$.

A function is called $\Sigma$-inner if it is $(-\Sigma)$-lossless. If $\Theta(z)$ is $\Sigma$-lossless, then $\Theta^{*}(z)$ is also $\Sigma$-lossless whereas both $\Theta^{\sharp}(z)$ and $\Theta(z)^{-1}$ are $\Sigma$-inner. The class of $\Sigma$-lossless functions is closed under multiplication.

To be consistent with the earlier definition of lossless functions, note that an $I_{k}$-lossless function is just called lossless. Likewise, an $I_{k}$-inner function is simply called inner. Throughout this paper we will be much concerned with lossless and inner functions of size $p \times p$, and with $J$-unitary and $J$-inner functions of size $2 p \times 2 p$, where $J$ denotes the following signature matrix:

$$
J=\left(\begin{array}{cc}
I_{p} & 0 \\
0 & -I_{p}
\end{array}\right) .
$$

Note that $J$-inner and $J$-lossless functions in general may have poles everywhere in the complex plane, but inner functions are analytic inside the unit disk and lossless functions are analytic outside the unit disk (including at the point at infinity). $J$-Lossless functions play a central role in the theory of chain scattering systems, see Kimura (1997).

\section{2 $\Sigma$-Balanced realizations of $\Sigma$-lossless matrix functions in discrete time}

In state-space, balanced realizations of discrete-time lossless systems are surprisingly easy to characterize. It is wellknown (cf., e.g., Hanzon et al. (2006) and the references given there) that $R=\left(\begin{array}{cc}D & C \\ B & A\end{array}\right)$ is a balanced realization matrix of a lossless system if and only if $R$ is a unitary matrix and $A$ is asymptotically stable. It then holds that the Gramians are given by $W_{c}=W_{o}=I_{n}$. If $R$ is unitary and $A$ has one or more eigenvalues on the unit circle, then the associated system is still lossless, but minimality does not hold.

Note that all balanced realizations of a lossless system are obtained from a given one, by applying unitary state-space 
transformations. The fact that all the Hankel singular values are identical to 1 makes that there is maximal freedom in choosing a balanced realization. This freedom can be used to build additional nice properties into the realization.

The following result applies to the more general class of discrete-time $\Sigma$-lossless systems. A proper $\Sigma$-lossless transfer function $G(z)$ of McMillan degree $n$ always admits a minimal $\Sigma$-balanced state-space realization $(A, B, C, D)$, i.e., one for which the realization matrix satisfies

$$
\left(\begin{array}{ll}
D & C \\
B & A
\end{array}\right)\left(\begin{array}{cc}
\Sigma & 0 \\
0 & I_{n}
\end{array}\right)\left(\begin{array}{ll}
D^{*} & B^{*} \\
C^{*} & A^{*}
\end{array}\right)=\left(\begin{array}{cc}
\Sigma & 0 \\
0 & I_{n}
\end{array}\right)
$$

Conversely, if this property holds, then $G(z)$ is proper $\Sigma$ lossless of McMillan degree $\leq n$. For a $\Sigma$-balanced statespace realization $(A, B, C, D)$ it holds that the solutions to the associated Lyapunov-Stein equations with modified right-hand sides $B \Sigma B^{*}$ and $C^{*} \Sigma C$, respectively, again satisfy $W_{c}=W_{o}=I_{n}$.

\subsection{J-unitary and J-inner functions}

Some particular $J$-inner functions that are intensively used in interpolation theory are the constant $J$-unitary matrices. We have the following result, for which details and proofs can be found in Dym (1989).

Theorem 1 . Every constant $J$-unitary matrix can be represented in a unique way as follows:

$$
M=H(E)\left(\begin{array}{cc}
P & 0 \\
0 & Q
\end{array}\right)
$$

where $P$ and $Q$ are $p \times p$ unitary matrices and $H(E)$ denotes the Halmos extension of a strictly contractive $p \times p$ matrix $E$ (i.e., such that $I-E^{*} E>0$ ). This Halmos extension $H(E)$ is defined by

$$
H(E)=\left(\begin{array}{cc}
\left(I-E E^{*}\right)^{-1 / 2} & E\left(I-E^{*} E\right)^{-1 / 2} \\
E^{*}\left(I-E E^{*}\right)^{-1 / 2} & \left(I-E^{*} E\right)^{-1 / 2}
\end{array}\right) .
$$

It holds that $H(E)$ is Hermitian, $J$-unitary and invertible with inverse $H(E)^{-1}=H(-E)$.

The important class of elementary $J$-inner factors (i.e., $J$ inner functions of McMillan degree one) is characterized by the following theorem.

Theorem 2. Let $\Theta(z)$ be an elementary $J$-inner factor with a pole at $z=1 / \bar{w}$. Then, apart from a constant $J$-unitary multiplier on the right, $\Theta(z)$ must be in one of the following three forms:

(1) If $w \in \mathbb{C},|w| \neq 1$

$$
\Theta(z)=I_{2 p}+\left(\frac{z-w}{1-\bar{w} z}-1\right) \frac{x x^{*} J}{x^{*} J x},
$$

for some vector $x \in \mathbb{C}^{2 p}$ such that $\frac{x^{*} J x}{1-|w|^{2}}>0$.

(2) If $|w|=1$,

$$
\Theta(z)=I_{2 p}+\delta \frac{(z+w)}{(z-w)} x x^{*} J
$$

for some nonzero $x \in \mathbb{C}^{2 p}$ such that $x^{*} J x=0$, and for some real $\delta>0$.

(3) If $w=\infty$, then

$$
\Theta(z)=I_{2 p}+\left(\frac{1}{z}-1\right) \frac{x x^{*} J}{x^{*} J x},
$$

for some $x \in \mathbb{C}^{2 p}$ such that $x^{*} J x<0$.

\subsection{The continuous-time case}

In the continuous-time case, the definition of a lossless matrix function is as follows.

Definition 3.3. In continuous-time, a square $p \times p$ rational matrix function $G(s)$ is called lossless (or stable all-pass) if at all points of analyticity it holds:

$$
\begin{array}{ll}
G(s)^{*} G(s) \geq I_{p}, & \text { for } \operatorname{Re}(s)<0, \\
G(s)^{*} G(s)=I_{p}, & \text { for } \operatorname{Re}(s)=0, \\
G(s)^{*} G(s) \leq I_{p}, & \text { for } \operatorname{Re}(s)>0 .
\end{array}
$$

The analogous extension to $\Sigma$-lossless functions $\Theta(s)$ now reads:

Definition 3.4. Let $\Sigma$ be a $k \times k$ signature matrix. Then a $k \times k$ rational matrix function $\Theta(z)$ is called continuoustime $\Sigma$-lossless if at at all points of analyticity it holds:

$$
\begin{array}{ll}
\Theta(s)^{*} \Sigma \Theta(s) \geq \Sigma, & \text { for } \operatorname{Re}(s)<0 \\
\Theta(s)^{*} \Sigma \Theta(s)=\Sigma, & \text { for } \operatorname{Re}(s)=0 \\
\Theta(s)^{*} \Sigma \Theta(s) \leq \Sigma, & \text { for } \operatorname{Re}(s)>0
\end{array}
$$

A balanced realization $(A, B, C, D)$ of a continuous-time $\Sigma$-lossless system is characterized by the following identities:

$$
\begin{aligned}
& A+A^{*}=-B \Sigma B^{*}, \\
& C=-D \Sigma B^{*}, \\
& D \Sigma D^{*}=\Sigma .
\end{aligned}
$$

Any proper $\Sigma$-lossless rational matrix function admits such a $\Sigma$-balanced realization. When the right-hand sides of the Lyapunov equations are modified to $-B \Sigma B^{*}$ and $-C^{*} \Sigma C$, respectively, it holds again that $W_{c}=W_{o}=I_{n}$. The relations for balanced realizations of lossless functions are simply obtained by setting $\Sigma$ to the identity matrix.

If the bilinear transform is applied to the $z$-domain, taking a $\Sigma$-lossless discrete-time function $\Theta(z)$ into a $\Sigma$ lossless continuous-time function $\widehat{\Theta}(s)=\Theta\left(\frac{z-1}{z+1}\right)$, then any associated $\Sigma$-balanced realization $(A, B, C, D)$ for $\Theta(z)$ is transformed into a $\Sigma$-balanced realization $(\widehat{A}, \widehat{B}, \widehat{C}, \widehat{D})$ for $\widehat{\Theta}(s)$ according to Eqns. (17)-(18).

3.5 Kronecker structures, triangular forms and truncation of the state vector

When choosing a balanced realization of a lossless system, one has the freedom of the unitary group to build in additional properties by means of state-space transformation. One property of interest is upper triangularity of the reachability matrix $K=\left(B A B \ldots A^{n-1} B\right)$, as upper triangularity is preserved under state vector truncation in a balance-and-truncate approach to model order reduction.

Generically, by applying QR-decomposition to $K$, a unitary state-space transformation matrix can be computed which makes the reachability matrix $K$ upper triangular while preserving balancedness. It depends on the Kronecker structure of the lossless system whether this is 
indeed possible. In such a case, the block-partitioned $n \times$ $(m+n)$ matrix $(B, A)$ is also upper triangular and state truncation preserves this property too.

As a result, state truncation for such a balanced realization of a continuous-time lossless system is easily seen to produce a balanced realization of a reduced order approximation system. In the discrete-time lossless case, balancedness is generally lost. It may be restored by reorthonormalization of the last $m$ columns of the truncated realization matrix, as balancedness requires a unitary realization matrix, but this affects the dynamical matrix and results in a definitely less attractive procedure than in the continuous-time case.

When dealing with any given Kronecker structure, a generalized procedure can be developed in which an associated suitable selection of $n$ columns of the reachability matrix $K$ is made upper triangular. This has been worked out in Peeters et al. (2007) using so-called nice selections, yielding a procedure which can be applied both in continuoustime and in discrete-time. This produces atlases of coordinate charts, exhibiting convenient triangularity properties which are preserved under state truncation, together covering the manifold of $p \times p$ lossless systems.

\section{INTERPOLATION THEORY FOR LOSSLESS SYSTEMS}

\subsection{Linear fractional transformations}

Linear fractional transformations occur extensively in representation formulas for the solution of various interpolation problems, see Ball et al. (1990). They are at the heart of the parametrization of lossless functions through the tangential Schur algorithm. The properties of a linear fractional transformation of matrices were studied in a form adapted to the needs of $J$-theory in Potapov (1988) and in an algebraic setting in Young (1984).

Let $\Theta \in \mathbb{C}^{2 p \times 2 p}(z)$ be an invertible rational matrix in $z$, block partitioned into four $p \times p$ blocks as $\Theta=\left(\begin{array}{cc}\Theta_{1} & \Theta_{2} \\ \Theta_{3} & \Theta_{4}\end{array}\right)$. Associated with $\Theta$, let the linear fractional transformation $\mathcal{T}_{\Theta}$ be defined to act on rational matrices $G \in \mathbb{C}^{p \times p}(z)$ in the following way:

$$
\mathcal{T}_{\Theta}: G \mapsto\left(\Theta_{4} G+\Theta_{3}\right)\left(\Theta_{2} G+\Theta_{1}\right)^{-1} .
$$

Then the following group property holds for the LFTs associated with two $2 p \times 2 p$ invertible matrices $\Phi$ and $\Psi$ :

$$
\mathcal{T}_{\Phi} \circ \mathcal{T}_{\Psi}=\mathcal{T}_{\Phi \Psi}
$$

Consequently, the inverse of $\mathcal{T}_{\Theta}$ is given by $\mathcal{T}_{\Theta}^{-1}=\mathcal{T}_{\Theta}$. It also holds that $\mathcal{T}_{\Phi}=\mathcal{T}_{\Psi}$ if and only if there exists a scalar function $\lambda \in \mathbb{C}(z)$ for which $\Phi=\lambda \Psi$. The latter still holds true, if the set of rational functions $G$ on which the LFTs $\mathcal{T}_{\Theta}$ act, is restricted to the real lossless functions; see Hanzon et al. (2006).

We have the following result, which explains the importance of LFTs with $J$-inner matrix functions for the construction of lossless functions.

Theorem 3. Let $\Theta$ be $J$-inner of size $2 p \times 2 p$ and of McMillan degree $m$. If $G$ is $p \times p$ lossless and of McMillan degree $n$, then the matrix function $\widehat{G}=\mathcal{T}_{\Theta}(G)$ is welldefined, lossless and of McMillan degree $\leq n+m$.
For a proof, see for instance (Ball et al., 1990, Sect. 18.2.).

If the $J$-inner matrix function $\Theta(z)$ happens to be constant (i.e., of McMillan degree 0), then the associated LFT $\mathcal{T}_{\Theta}(G)$ does not affect the McMillan degree of the lossless function $G$.

\subsection{The tangential Schur algorithm}

In Ober (1987b); Hanzon and Ober (1998) balanced statespace canonical forms have been constructed for various classes of linear systems, with special properties of these classes (such as stability) built in. A balanced canonical form for continuous-time SISO lossless systems was presented in Ober (1987a). This continuous-time lossless case is central to the constructions of Hanzon and Ober (1997), Hanzon and Ober (1998). In the scalar case, it is balanced with a positive upper triangular reachability matrix, while Kronecker indices and nice selections are used in the multivariable case to arrive at balanced overlapping canonical forms.

For discrete-time lossless systems, application of the bilinear transform preserves balancedness, but it destroys positive upper triangularity of the reachability matrix, which is unattractive for balance-and-truncate type procedures. Therefore, in Hanzon and Peeters (2000) a balanced canonical form for discrete-time lossless SISO systems was developed directly, which is parameterized by Schur parameters and which yields an upper triangular reachability matrix $K$. The unitary realization matrix $R$ is factored into a product of elementary unitary matrices, thus facilitating a recursive construction of the balanced canonical form. This SISO balanced canonical form was generalized to the MIMO case in Hanzon et al. (2006), using linear fractional transformations and the tangential Schur algorithm.

The tangential Schur algorithm yields a flexible approach to the recursive construction of a parametrization of the space of lossless systems of a given order $n$. It is derived from the method of Alpay et al. (1994); Fulcheri and Olivi (1998), where the tangential Schur algorithm is used to construct an infinite atlas of generic overlapping parameterizations for the space of $p \times p$ inner functions of McMillan degree $n$. The relationship between these two situations is constituted by the map $R(z) \mapsto R^{\sharp}(z)$, which relates $p \times p$ inner functions of McMillan degree $n$ to $p \times p$ lossless functions of McMillan degree $n$.

In the context of inner functions, the tangential Schur algorithm consists of an iterative procedure by which a given $p \times p$ inner function of McMillan degree $n$ is reduced in $n$ iteration steps to a $p \times p$ inner function of McMillan degree 0 (i.e., to a constant unitary matrix). In each iteration step the McMillan degree of the inner function at hand is reduced by 1 , by application of a suitable linear fractional transformation which is chosen to meet a particular interpolation condition, and which involves an associated $J$-inner matrix function of McMillan degree 1. The actual parametrization procedure consists of the reverse process, by which a chart of inner functions of McMillan degree $n$ is constructed in $n$ iteration steps, starting from an initial unitary matrix. The choice of interpolation points and (normalized) direction vectors 
may serve to index such a chart, while the local coordinates correspond to the $n$ Schur vectors.

In the general form of the tangential Schur algorithm discussed in Hanzon et al. (2006), each elementary $J$-inner factor has its pole outside the closed unit disk at $z=1 / \bar{w}$. It can be represented as:

$$
\begin{aligned}
& \Theta_{u, v, w, \xi, H}(z)= \\
& =\left(I_{2 p}+\left(\frac{(z-w)(1-\bar{w} \xi)}{(1-\bar{w} z)(\xi-w)}-1\right) \frac{\left(\begin{array}{l}
u \\
v
\end{array}\right)\left(\begin{array}{l}
u \\
v
\end{array}\right)^{*} J}{\left(1-\|v\|^{2}\right)}\right) H
\end{aligned}
$$

where

(1) $w \in \mathbb{C},|w|<1$, (the interpolation point),

(2) $u \in \mathbb{C}^{p \times 1},\|u\|=1$, (the normalized direction vector),

(3) $v \in \mathbb{C}^{p \times 1},\|v\|<1$, (the Schur parameter vector),

(4) $\xi \in \mathbb{C},|\xi|=1$, (a normalization point on the circle),

(5) and $H$ is a $2 p \times 2 p$ constant $J$-unitary matrix.

This representation is of the form (1) in Thm. 2, as seen by imposing: $x=\left(\begin{array}{c}u \\ v\end{array}\right)$, with $\|u\|=1$ and thus $\|v\|<1$; the right $J$-unitary factor $H$ is chosen as $H=\Theta(\xi)$.

The following proposition further clarifies the structure of $\Theta_{u, v, w, \xi, H}(z)$ and of the action of the associated LFT:

Proposition 4. The $J$-inner matrix function $\Theta_{u, v, w, \xi, H}(z)$ can be factored as:

$$
\Theta_{u, v, w, \xi, H}(z)=H\left(u v^{*}\right) S_{u, w}(z) S_{u, w}(\xi)^{-1} H\left(u v^{*}\right)^{-1} H
$$

where $H\left(u v^{*}\right)$ denotes the Halmos extension of the strictly contractive matrix $u v^{*}$, and where

$$
S_{u, w}(z):=\left(\begin{array}{cc}
I_{p}+\left(b_{w}(z)-1\right) u u^{*} & 0 \\
0 & I_{p}
\end{array}\right),
$$

with $b_{w}(z)=\frac{z-w}{1-\bar{w} z}$.

Recall that an LFT involving a product of matrices comes down to a composition of LFTs involving each of the individual factors. Also, an LFT involving a constant $J$ unitary matrix does not change the McMillan degree. Therefore, only the LFT involving the factor $S_{u, w}(z)$ can affect the McMillan degree. The block-diagonal structure of $S_{u, w}(z)$ implies that for this LFT postmultiplication takes place by $\left(I_{p}+\left(b_{w}(z)-1\right) u u^{*}\right)^{-1}=$ $I_{p}+\left(\frac{1-\bar{w} z}{z-w}-1\right) u u^{*}$. The next proposition (see Hanzon et al. (2006)) establishes the effect of the LFT associated with $\Theta_{u, v, w, \xi, H}(z)$ on a lossless function $G(z)$ of McMillan degree $n$.

Proposition 5. Let $G(z)$ be a lossless function of McMillan degree $n$. Then

$$
\widehat{G}(z)=\mathcal{T}_{\Theta_{u, v, w, \xi, H}(z)}(G(z))
$$

is lossless of McMillan degree $n+1$ and satisfies the interpolation condition

$$
\widehat{G}(1 / \bar{w}) u=v
$$

The reverse process is addressed by the following proposition. It describes the construction of a lossless matrix function $G(z)$ of reduced degree $n$ from a given lossless matrix function $\widehat{G}(z)$ of McMillan degree $n+1$ proceeding from an interpolation condition of the form (48). See also, e.g., Ball et al. (1990); Alpay et al. (1994); Hanzon et al. (2006).

Proposition 6 . Let $\widehat{G}$ be a $p \times p$ lossless function of McMillan degree $n+1$ which satisfies an interpolation condition of the form

$$
\widehat{G}(1 / \bar{w}) u=v,
$$

in which $w \in \mathbb{C}$ is an interpolation point with $|w|<1$, $u \in \mathbb{C}^{p \times 1}$ is a normalized direction vector with $\|u\|=1$ and $v \in \mathbb{C}^{p \times 1}$ is a Schur vector satisfying $\|v\|<1$.

Let $\xi \in \mathbb{C}$ be an arbitrary number of modulus 1 , and let $H$ be an arbitrary constant $J$-unitary matrix. Then $\widehat{G}$ admits the representation

$$
\widehat{G}=\mathcal{T}_{\Theta_{u, v, w, \xi, H}}(G),
$$

for some $p \times p$ lossless function $G$ of McMillan degree $n$.

Note that in the standard case with $w=0$ the value of $\widehat{G}(\infty)$ corresponds to the direct feedthrough term $\widehat{D}$ of any state-space realization $(\widehat{A}, \widehat{B}, \widehat{C}, \widehat{D})$ of $\widehat{G}(z)$, so that the interpolation condition then takes the form $\widehat{D} u=v$. This case is often encountered when constructing canonical forms with additional triangularity properties.

The tangential Schur algorithm can now be described as follows.

Theorem 7. (Tangential Schur algorithm). Let $G^{(n)}$ be a $p \times p$ lossless transfer matrix of McMillan degree $n$. For $k=n, \ldots, 1$, let interpolation points $w_{k} \in \mathbb{C}$ be given with $\left|w_{k}\right|<1$, let constants $\xi_{k} \in \mathbb{C}$ be given with $\left|\xi_{k}\right|=1$, and let mappings $H_{k}:(u, v, w, \xi) \mapsto H_{k}(u, v, w, \xi)$ be given which assign a constant $2 p \times 2 p J$-unitary matrix $H_{k}(u, v, w, \xi)$ to each quadruple $(u, v, w, \xi) \in \mathbb{C}^{p} \times \mathbb{C}^{p} \times$ $\mathbb{C} \times \mathbb{C}$ with $\|u\|=1,\|v\|<1,|w|<1$ and $|\xi|=1$.

Then for $k=n, \ldots, 1$, there exist vectors $u_{k} \in \mathbb{C}^{p}$ with $\left\|u_{k}\right\|=1$, such that the vectors $v_{k} \in \mathbb{C}^{p}$ constructed recursively by the following formulas, each have length $\left\|v_{k}\right\|<1$ :

$$
\begin{aligned}
& v_{k}:=G^{(k)}\left(1 / \bar{w}_{k}\right) u_{k}, \\
& \Theta_{k}:=\Theta\left(u_{k}, v_{k}, w_{k}, \xi_{k}, H_{k}\left(u_{k}, v_{k}, w_{k}, \xi_{k}\right)\right), \\
& G^{(k-1)}:=\mathcal{T}_{\Theta_{k}}^{-1}\left(G^{(k)}\right) .
\end{aligned}
$$

With such a choice of the unit vectors $u_{k}(k=n, \ldots, 1)$ each of the functions $G^{(k)}$ is lossless of McMillan degree $k$ and one can write

$$
\begin{aligned}
& G^{(n)}=\mathcal{T}_{\Theta_{n}}\left(\mathcal{T}_{\Theta_{n-1}}\left(\ldots\left(\mathcal{T}_{\Theta_{1}}\left(G^{(0)}\right)\right) \ldots\right)\right)= \\
& =\mathcal{T}_{\Theta_{n} \Theta_{n-1} \ldots \Theta_{1}}\left(G^{(0)}\right),
\end{aligned}
$$

where $G^{(0)}$ is a constant unitary matrix.

The tangential Schur algorithm offers a recursive way to reduce a given $p \times p$ lossless matrix function of McMillan degree $n$ to a unitary constant matrix $G^{(0)}$. In each of the $n$ steps the McMillan degree is decreased by one. Each step is associated with an interpolation condition. The reversed tangential Schur algorithm can be used to generate a parameterized chart of lossless systems, in which the Schur vectors $v_{1}, v_{2}, \ldots, v_{n}$ and the unitary matrix $G^{(0)}$ serve as the parameters. The set of values for $w_{k}, u_{k}, \xi_{k}$ and the set of mappings $H_{k}$ at the $n$ recursion steps can serve to index 
a generic chart for the differentiable manifold of lossless functions of degree $n$, if the mappings $H_{k}$ are sufficiently smooth. Such a chart involves $n p+\frac{1}{2} p(p-1)$ real degrees of freedom in the real case, and $2 n p+p^{2}$ real degrees of freedom in the complex case.

\subsection{State-space realizations}

In Hanzon et al. (2006) it is established under which conditions a recursion step of the (reversed) tangential Schur algorithm can be implemented on the state-space realization level by means of straightforward unitary matrix multiplication of the realization matrix $R$. More precisely, we first introduce the following definition of a mapping $\mathcal{F}_{U, V}$.

Definition 4.1. For any two matrices $U$ and $V$ of size $(p+$ $1) \times(p+1)$, the associated mapping $\mathcal{F}_{U, V}$ is defined to act on a proper rational $p \times p$ matrix function $G(z)$ as follows:

$$
\mathcal{F}_{U, V}: G(z) \mapsto F_{1}(z)+\frac{F_{2}(z) F_{3}(z)}{z-F_{4}(z)},
$$

with $F_{1}(z)$ of size $p \times p, F_{2}(z)$ of size $p \times 1, F_{3}(z)$ of size $1 \times p$ and $F_{4}(z)$ scalar, defined by:

$$
F(z)=\left(\begin{array}{ll}
F_{1}(z) & F_{2}(z) \\
F_{3}(z) & F_{4}(z)
\end{array}\right)=V\left(\begin{array}{cc}
1 & 0 \\
0 & G(z)
\end{array}\right) U^{*}
$$

Then the next result establishes that a state-space realization of $\widetilde{G}(z)=\mathcal{F}_{U, V}(G(z))$ can in fact be obtained by operating directly on an available realization matrix $R$ of $G(z)$ through pre- and post-multiplication by the matrices $V$ and $U^{*}$.

Proposition 8. Let $U$ and $V$ be two matrices of size $(p+$ $1) \times(p+1)$. Let $G(z)$ be a $p \times p$ proper rational transfer function admitting a state-space realization $(A, B, C, D)$. Then a state-space realization $(\widetilde{A}, \widetilde{B}, \widetilde{C}, \widetilde{D})$ of $\widetilde{G}(z)=$ $\mathcal{F}_{U, V}(G(z))$ with $(n+1)$-dimensional state-space is given by:

$$
\left(\begin{array}{cc}
\widetilde{D} & \widetilde{C} \\
\widetilde{B} & \widetilde{A}
\end{array}\right)=\left(\begin{array}{cc}
V & 0 \\
0 & I_{n}
\end{array}\right)\left(\begin{array}{lll}
1 & 0 & 0 \\
0 & D & C \\
0 & B & A
\end{array}\right)\left(\begin{array}{cc}
U^{*} & 0 \\
0 & I_{n}
\end{array}\right)
$$

Clearly, if we are dealing with a balanced realization of a lossless function $G(z)$, then the realization matrix $R$ is unitary and pre- and post-multiplication with unitary matrices $V$ and $U^{*}$ preserves this property, so that $\widetilde{G}(z)$ is again lossless and the state-space realization $(\widetilde{A}, \widetilde{B}, \widetilde{C}, \widetilde{D})$ is balanced if and only if $\widetilde{A}$ is asymptotically stable.

When employing a linear fractional transformation involving an elementary $J$-inner factor $\Theta_{u, v, w, \xi, H}(z)$, we have the following major result.

Theorem 9. If $\mathcal{T}_{\Theta_{u, v, w, \xi, H}(z)}$ coincides with a mapping $\mathcal{F}_{U, V}$ with $U$ and $V$ unitary, then

$$
\Theta_{u, v, w, \xi, H}(z)=H\left(u v^{*}\right) S_{u, w}(z) H\left(\bar{w} u v^{*}\right)\left(\begin{array}{cc}
P & 0 \\
0 & Q
\end{array}\right),
$$

for some $u, v \in \mathbb{C}^{p}$ with $\|u\|=1,\|v\|<1$, some $w \in \mathbb{C}$ with $|w|<1$, and some $p \times p$ unitary matrices $P$ and $Q$. Equivalently it holds that

$$
H=H\left(u v^{*}\right) S_{u, w}(\xi) H\left(\bar{w} u v^{*}\right)\left(\begin{array}{cc}
P & 0 \\
0 & Q
\end{array}\right) .
$$

In that case one can take $U=\widehat{U}\left(\begin{array}{ll}1 & 0 \\ 0 & P\end{array}\right)$ and $V=$ $\widehat{V}\left(\begin{array}{ll}1 & 0 \\ 0 & Q\end{array}\right)$, where

$$
\begin{aligned}
& \widehat{U}=\left(\begin{array}{cc}
\frac{\sqrt{1-|w|^{2}}}{\sqrt{1-|w|^{2}|| v \|^{2}}} u & I_{p}-\left(1+\frac{w \sqrt{1-\|v\|^{2}}}{\sqrt{1-|w|^{2}\|v\|^{2}}}\right) u u^{*} \\
\frac{\bar{w} \sqrt{1-\|v\|^{2}}}{\sqrt{1-|w|^{2}\|v\|^{2}}} & \frac{\sqrt{1-|w|^{2}}}{\sqrt{1-|w|^{2}\|v\|^{2}}} u^{*}
\end{array}\right)(60) \\
& \widehat{V}=\left(\begin{array}{cc}
\frac{\sqrt{1-|w|^{2}}}{\sqrt{1-|w|^{2}\|v\|^{2}}} v & I_{p}-\left(1-\frac{\sqrt{1-\|v\|^{2}}}{\sqrt{1-|w|^{2}\|v\|^{2}}}\right) \frac{v v^{*}}{\|v\|^{2}} \\
\frac{\sqrt{1-\|v\|^{2}}}{\sqrt{1-|w|^{2}\|v\|^{2}}} & -\frac{\sqrt{1-|w|^{2}}}{\sqrt{1-|w|^{2}\|v\|^{2}}} v^{*}
\end{array}\right)(61)
\end{aligned}
$$

For the special case $w=0$ it follows that

$$
\begin{aligned}
\widehat{U} & =\left(\begin{array}{cc}
u & I_{p}-u u^{*} \\
0 & u^{*}
\end{array}\right) \\
\widehat{V} & =\left(\begin{array}{cc}
v & I_{p}-\left(1-\sqrt{1-\|v\|^{2}}\right) \frac{v v^{*}}{\|v\|^{2}} \\
\sqrt{1-\|v\|^{2}} & -v^{*}
\end{array}\right) .
\end{aligned}
$$

In the SISO case (i.e.: $p=1$ ) it is natural to choose $u=1$ and $\widehat{U}=I_{2}$. Then $\widehat{V}=\left(\begin{array}{cc}v & \sqrt{1-\|v\|^{2}} \\ \sqrt{1-\|v\|^{2}} & -v^{*}\end{array}\right)$. It follows that the realization matrix $R$ attains a positive upper-Hessenberg form, for which the reachability matrix is positive upper triangular; this yields the canonical form presented in Hanzon and Peeters (2000). In the MIMO case, when $u$ is chosen to be a standard basis vector, the matrix $\widehat{U}$ becomes a permutation matrix. This situation is further investigated in Peeters et al. (2007), where it is established which choices of standard basis vectors in the consecutive recursion steps are required to yield reachability matrices with particular pivot structures, corresponding to prescribed Kronecker structures. In Hanzon et al. (2009) it is shown that all choices of standard basis vectors produce subdiagonal canonical forms.

If the interpolation point $w$ is chosen to be a pole of the lossless function $G(z)$ and if $u$ is chosen to be a normalized vector in the kernel of $G\left(\bar{w}^{-1}\right)$, it follows that the Schur vector attains the value $v=0$. Repeating this procedure for each step of the tangential Schur algorithm produces the Potapov factorization of $G(z)$, i.e.: $G(z)$ is factored into a product of lossless functions of degree 1 . This is a useful property, which is used in the software package RARL2, see Olivi and Marmorat (2003), to change the local parameterization whenever the current parameterization is considered to become less well conditioned.

If $G(z)$ is real, it is straightforward to arrive at a real parameterization by requiring the interpolation points, direction vectors, and so on, all to be real.

In another line of research, presented in Peeters et al. (2001), it was investigated how an available state-space realization of a proper $J$-lossless matrix $\Theta\left(z^{-1}\right)$ and a statespace realization of a lossless function $G(z)$ can jointly be employed to directly yield a state-space realization of the lossless function $\widehat{G}(z)=\mathcal{T}_{\Theta(z)}$ in the form of an LFT acting on an extended realization matrix. When the realization for $\Theta\left(z^{-1}\right)$ is $J$-balanced and the realization for $G(z)$ is 
balanced, then the realization thus obtained for $\widehat{G}(z)$ is again balanced.

Theorem 10. Let $\Theta(z)=\left(\begin{array}{cc}\Theta_{1}(z) & \Theta_{2}(z) \\ \Theta_{3}(z) & \Theta_{4}(z)\end{array}\right)$ be $J$-inner of McMillan degree $m$. Suppose that the $J$-lossless function $\Theta\left(z^{-1}\right)$ is proper, for which a $J$-balanced state-space realization is given by $\left(\mathcal{A},\left(\begin{array}{c}\mathcal{B}_{1} \\ \mathcal{B}_{2}\end{array}\right),\left(\begin{array}{ll}\mathcal{C}_{1} & \mathcal{C}_{2}\end{array}\right),\left(\begin{array}{cc}\mathcal{D}_{1} & \mathcal{D}_{2} \\ \mathcal{D}_{3} & \mathcal{D}_{4}\end{array}\right)\right)$.

Let $G(z)$ be lossless of McMillan degree $n$, and suppose that a balanced realization is given by $(A, B, C, D)$, which gives rise to an extended realization matrix $R_{e}$ given by

$$
R_{e}=\left(\begin{array}{ccc}
D & 0 & C \\
0 & I_{m} & 0 \\
B & 0 & A
\end{array}\right)
$$

Let $\Delta$ be defined in terms of the partitioned $J$-balanced realization of $\Theta\left(z^{-1}\right)$ by:

$$
\Delta=\left(\begin{array}{cccccc}
\mathcal{D}_{1} & \mathcal{C}_{1} & 0 & \mathcal{D}_{2} & 0 & 0 \\
\mathcal{B}_{1} & \mathcal{A} & 0 & \mathcal{B}_{2} & 0 & 0 \\
0 & 0 & I_{n} & 0 & 0 & 0 \\
\mathcal{D}_{3} & \mathcal{C}_{2} & 0 & \mathcal{D}_{4} & 0 & 0 \\
0 & 0 & 0 & 0 & I_{m} & 0 \\
0 & 0 & 0 & 0 & 0 & I_{n}
\end{array}\right)
$$

Then a balanced realization $(\widehat{A}, \widehat{B}, \widehat{C}, \widehat{D})$ for the lossless function $\widehat{G}(z)=\mathcal{T}_{\Theta(z)}$ is given by the LFT

$$
\widehat{R}=\left(\begin{array}{ll}
\widehat{D} & \widehat{C} \\
\widehat{B} & \widehat{A}
\end{array}\right)=\mathcal{T}_{\Delta}\left(R_{e}\right) .
$$

When restricting to elementary $J$-inner factors $\Theta(z)$, however, note that this procedure generates alternative balanced state-space realizations, not previously produced by the realization procedures presented earlier.

\subsection{Boundary interpolation}

Above it was shown that upper triangular structures in the reachability matrix are conveniently obtained in discretetime upon choosing the interpolation points $w$ at zero and the direction vectors $u$ from the set of standard basis vectors in a suitable way. Application of the bilinear transform takes these realizations into balanced realizations for continuous-time lossless systems. However, this approach does not allow one to build positive upper triangularity into the continuous-time reachability matrix.

In Hanzon et al. (2008) it was established for the SISO case that positive upper triangularity of the reachability matrix in continuous-time (as exhibited by Ober's canonical form, see Ober $(1987 \mathrm{~b}, \mathrm{a})$ ) does in fact correspond to choosing the interpolation points $w$ at infinity. For the associated discrete-time case (related by the bilinear transform) the elementary $J$-inner factor $\Theta(z)$ is of the form (2), with $w$ chosen on the unit circle at $w=-1$.

In discrete-time the corresponding boundary interpolation problem not only involves a condition of the form $G\left(\bar{w}^{-1}\right) u=v$ with $\|u\|=1$, but also a second condition of the form $v^{*} G^{\prime}\left(\bar{w}^{-1}\right) u=-w^{-1} \rho$, with a real positive value for $\rho$. At $w=-1$ these conditions take the form

$$
\begin{aligned}
& G(-1) u=v, \\
& v^{*} G^{\prime}(-1) u=\rho .
\end{aligned}
$$

The fact that $G(z)$ is unitary for each point on the unit circle makes that $\|v\|=1$, thus leaving one (real) degree of freedom. The property that $\rho$ is real and positive, stems from the fact that for a lossless function $G(z)$, the matrix $-z G(z)^{*} G^{\prime}(z)$ is positive semi-definite for all $z$ on the unit circle (and $u$ can always be chosen such that positivity of $\rho$ holds, provided that the degree of $G(z)$ is at least 1$)$. See for instance (Ball et al., 1990, Ch. 21) for a treatment of such boundary interpolation problems.

To apply this theory to the case at hand, note that it is inconvenient to address the derivative of a continuous-time lossless function $G(s)$ at $s=\infty$ directly. Instead, a useful way to proceed is by studying the related function $G\left(s^{-1}\right)$ and its derivatives at zero. This approach has been worked out in Hanzon et al. (2008).

For the continuous-time lossless MIMO case, positive upper triangularity can also be built into the reachability matrix of a balanced realization, by generalizing the results of Hanzon et al. (2008). These recent results will appear elsewhere.

\section{APPLICATIONS}

\subsection{Parameterization of stable systems: input normal} forms

The parameterizations presented above have been developed primarily for lossless systems in discrete-time or in continuous-time. However, they are easily employed for parameterizing the class of stable systems of a given order $n$ too; see also Hanzon and Ober (1998). To this end, observe that a stable system can generically be brought into canonical form by first making the system input normal (i.e., the reachability Gramian $W_{c}$ equals the identity matrix $I_{n}$ ) and then using orthogonal state-space transformations (which leave input normality unaffected) to make the reachability matrix $K$ positive upper triangular. Obviously, instead of input normality and a positive upper triangular reachability matrix, one may also concentrate on output normality and a positive lower triangular observability matrix. In the MIMO case, Kronecker indices and nice selections can be used to develop a canonical form along similar lines (see Peeters et al. (2007)).

In terms of realization matrices, it suffices to parameterize the part involving the input normal pair $(A, B)$ and then to supply arbitrary matrices $C$ and $D$, with $C$ such that observability holds. Here it is helpful to notice that without loss of generality the reversed tangential Schur algorithm can be started from $G^{(0)}=I_{p}$. Thus, the parameterization of all input normal pairs $(A, B)$ for which $K$ is positive lower triangular only involves the sequence of Schur vectors.

\subsection{System identification: separable least squares}

In linear system identification, the method of separable least squares (SLS) was first introduced in Golub and Pereyra (1973). In Bruls et al. (1999) one proceeds by reducing the nonlinear least squares optimization problem at hand, to a new optimization problem over the space of output normal pairs $(C, A)$. In this approach, the matrices $B$ and $D$ are analytically optimized for the original criterion 
function in terms of $A$ and $C$, and subsequently eliminated from the problem. As we have seen in the previous subsection, output normal pairs $(C, A)$ are intimately related to lossless systems satisfying a normalization condition, and they can be conveniently parameterized with Schur vectors. The state-space approach discussed in the present paper allows one to proceed in the discrete-time case with parameterizations which are constructed as a product of unitary matrices.

\section{$5.3 L_{2}$-System approximation and $R A R L 2$}

In $L_{2}$-system approximation, the Douglas-Shapiro-Shields factorization, see Douglas et al. (1970), can be used to factor a given strictly proper stable rational transfer matrix function $H$ into a product $G P$ in which $G$ is lossless of the same McMillan degree as $H$ and $P$ is unstable. The factor $G$ is unique up to a right unitary factor. The $L_{2}$ approximation problem can subsequently be reformulated, analytically, entirely in terms of $G$. Parameterization of the class of lossless systems according to the state-space methods described here, allows one to handle the stability constraint conveniently, by optimizing over the class of lossless systems.

In Marmorat et al. (2002) this approach was worked out both in discrete-time and in continuous-time, using the balanced state-space parameterizations discussed above. Later, the procedure was implemented in the Matlab software package RARL2, see Olivi and Marmorat (2003), which employs a chart switching strategy to move to the center of a new parameter chart when the current approximation moves too far away from the center of the current chart. This employs the fact that each lossless system can be put at the center of an adapted chart, where all the Schur vectors $v_{k}$ are zero, by means of the Potapov factorization. The distance to the center of a chart is intuitively measured by the lengths of the Schur vectors used to represent the lossless system in that chart: when the length of a Schur vector becomes equal to 1 , the boundary of a chart is reached and degeneration phenomena may occur.

We find it interesting to note that the numerical conditioning of the implemented computational procedure is considerably better for the balanced state-space realizations in discrete-time with their associated unitary matrix factorizations, when compared to an earlier implementation which performed analogous computations directly on the level of transfer functions (see Fulcheri and Olivi (1998)).

\subsection{Orthogonal wavelets from filter banks}

In modern digital signal processing, an important class of wavelets is constituted by the orthogonal wavelets that are generated by power complementary filter banks. In Strang and Nguyen (1996) a detailed exposition of this topic is given. In its classical form, the procedure is as follows. First, two copies of the same discrete-time input signal are passed through a lowpass filter $C(z)$ and a highpass filter $D(z)$, to produce approximation coefficients and detail coefficients, respectively. These output sequences are then downsampled by a factor 2, i.e., only the even-indexed values (the even phases of the signals) are stored. When the filters $C(z)$ and $D(z)$ are power complementary (see also Vaidyanathan and Doğanata (1989)), no information loss occurs and the output sequences admit an interpretation in terms of associated orthonormal bases, spanned by the socalled scaling function (used for approximation purposes) and the wavelet function (used to analyze the details). One then proceeds repeatedly, in a likewise fashion, by processing the approximation signal (the downsampled output of the filter $C(z))$ to compute approximation coefficients and detail coefficients on coarser scales.

For each level of the analysis, an equivalent procedure can also be considered, in which the input signal is first split into its two phases (the even and odd phases), which are then jointly processed by a $2 \times 2$ polyphase filter $H(z)$ to produce the same (downsampled) sequences of approximation and detail coefficients as before. It then follows that orthonormality of the wavelet bases, and power complementarity of the filters $C(z)$ and $D(z)$, are in fact equivalent to $H(z)$ being (discrete-time) lossless. The theory presented here for the construction of parameterizations of lossless systems can thus be applied to facilitate parametric optimization over the class of lossless $2 \times 2$ matrix functions of a given degree $n$. This is particularly useful for wavelet design, especially when a wavelet is designed for a specific application. Initial investigations using this approach are presented in Karel et al. (2005).

Extensions to more complicated situations are currently under investigation. One issue of practical interest concerns the number of vanishing moments that a wavelet has. As it turns out, the problem of building in vanishing moments can be reformulated as an interpolation problem for $2 \times 2$ lossless systems on the stability boundary (at $z=1$ ) involving higher order derivatives. Another obvious extension concerns multiwavelets, where the polyphase matrix is generalized to become a lossless matrix of size $2 r \times 2 r$ with $r>1$; see also Peeters et al. (2006).

\section{CONCLUSIONS}

In this paper we have presented an overview of the main results which link balanced parameterizations of lossless systems to interpolation theory and Schur parameters in various settings: discrete-time and continuous-time, SISO and MIMO. It is indicated which choices in the tangential Schur algorithm (in particular which choice of interpolation points) supports the construction of triangular pivot structures in reachability matrix. It is possible to deal with special Kronecker structures and restrictions to important subclasses (such real lossless systems) are conveniently handled. Certain extensions of the presented theory, not discussed here, are also available in the literature. Most notably these involve Nudelman interpolation problems and symmetric inner functions. Further lines of research concern the application domains indicated above, which often present specific additional constraints that require special attention.

\section{REFERENCES}

Alpay, D., Baratchart, L., and Gombani, A. (1994). On the differential structure of matrix-valued rational inner functions. Operator Theory: Advances and Applications, $73,30-66$. 
Ball, J., Gohberg, I., and Rodman, L. (1990). Interpolation of rational matrix functions, volume 45 of Operator Theory: Advances and Applications. Birkhäuser.

Baratchart, L. (1990). On the topological structure of inner functions and its use in identification, volume 8, 51-59. Birkhäuser.

Bruls, J., Chou, C., Haverkamp, B., and Verhaegen, M. (1999). Linear and non-linear system identification using separable least-squares. European Journal of Control, 5(1), 116-128.

Douglas, R., Shapiro, H., and Shields, A. (1970). Cyclic vectors and invariant subspaces for the backward shift operator. Annales de l'Institut Fourier (Grenoble), 20, 37-76.

Dym, H. (1989). J-contractive matrix functions, reproducing kernel spaces and interpolation, volume 71 of $C B M S$ lecture notes. American Mathematical Society, Rhodes Island.

Fulcheri, P. and Olivi, M. (1998). Matrix rational $H^{2}$ approximation: A gradient algorithm based on Schur analysis. SIAM Journal on Control and Optimization, 36(6), 2103-2127.

Genin, Y., Van Dooren, P., Kailath, T., Delosme, J., and Morf, M. (1983). On $\Sigma$-lossless transfer functions and related questions. Linear Algebra and its Applications, 50, 251-275.

Glover, K. (1984). All optimal Hankel-norm approximations of linear multivariable systems and their $L_{\infty}$-error bounds. International Journal of Control, 39(6), 11151193.

Golub, G. and Pereyra, V. (1973). The differentiation of pseudoinverse and nonlinear least squares problems whose variables separate. SIAM Journal of Numerical Analysis, 10(2), 413-432.

Hanzon, B. and Ober, R. (1997). Overlapping blockbalanced canonical forms and parametrizations: the SISO case. SIAM Journal of Control and Optimization, 35, 228-242.

Hanzon, B. and Ober, R. (1998). Overlapping blockbalanced canonical forms for various classes of linear systems. Linear Algebra and its Applications, 281, 171225

Hanzon, B., Olivi, M., and Peeters, R. (2006). Balanced realizations of discrete-time stable all-pass systems and the tangential Schur algorithm. Linear Algebra and Applications, 418, 793-820.

Hanzon, B., Olivi, M., and Peeters, R. (2008). Lossless scalar functions: Boundary interpolation, Schur algorithm and Ober's canonical form. In Proceedings of the 47th Conference on Decision and Control, Cancun, $1845-1850$

Hanzon, B., Olivi, M., and Peeters, R. (2009). Subdiagonal pivot structures and associated canonical forms under state isometries. In Proceedings of SYSID 2009, St. Malo.

Hanzon, B. and Peeters, R. (2000). Balanced parametrizations of stable SISO all-pass systems in discrete-time. Mathematics of Control, Signals, and Systems, 13, 240276.

Kailath, T. (1980). Linear Systems. Prentice-Hall.

Karel, J., Peeters, R., Westra, R., Moermans, K., Haddad, S., and Serdijn, W. (2005). Optimal discrete wavelet design for cardiac signal processing. In Proceedings of the 27th IEEE EMBS Annual International Conference (EMBC 2005), Shanghai, 2769-2772.

Kimura, H. (1997). Chain-Scattering Approach to $H^{\infty}$ Control. Birkhäuser.

Leblond, J. and Olivi, M. (1998). Weighted $H^{2}$ approximation of transfer functions. Mathematics of Control, Signals, and Systems, 11, 28-39.

Marmorat, J., Olivi, M., Hanzon, B., and Peeters, R. (2002). Matrix rational $H^{2}$-approximation: a statespace approach using Schur parameters. In Proceedings of the 41st Conference on Decision and Control, Las Vegas, 4244-4249.

Moore, B. (1981). Principal component analysis in linear systems: Controllability, observability, and model reduction. IEEE Transactions on Automatic Control, AC-26, $17-32$.

Ober, R. (1987a). Asymptotically stable allpass transfer functions: canonical form, parametrization and realization. In Proceedings of the IFAC World Congress, Munich.

Ober, R. (1987b). Balanced realizations: canonical form, parametrization, model reduction. International Journal of Control, 46, 643-670.

Olivi, M. and Marmorat, J. (2003). RARL2, Matlab based software package developed by Team APICS at INRIA, Sophia-Antipolis, France. Available at: http: //www-sop.inria.fr/apics/RARL2/rarl2.html.

Peeters, R., Hanzon, B., and Olivi, M. (2001). Linear fractional transformations and balanced realization of discrete-time stable all-pass systems. In Proceedings of the 1st IFAC Symposium on System Structure and Control, Prague.

Peeters, R., Hanzon, B., and Olivi, M. (2007). Canonical lossless state-space systems: Staircase forms and the Schur algorithm. Linear Algebra and Applications, 425, 404-433.

Peeters, R., Karel, J., Westra, R., Haddad, S., and Serdijn, W. (2006). Multiwavelet design for cardiac signal processing. In Proceedings of the 28th IEEE EMBS Annual International Conference (EMBC 2006), New York City, 1682-1685.

Pernebo, L. and Silverman, L. (1982). Model reduction via balanced state space representations. IEEE Transactions on Automatic Control, AC-27, 382-387.

Potapov, V. (1960). The multiplicative structure of $J$ contractive matrix functions. Amer. Math. Soc. Transl. (2), 15, 131-243.

Potapov, V. (1988). Linear fractional transformations of matrices. Amer. Math. Soc. Transl. (2), 138, 21-35.

Strang, G. and Nguyen, T. (1996). Wavelets from Filter Banks. Cambridge-Wellesley.

Vaidyanathan, P. and Doğanata, Z. (1989). The role of lossless systems in modern digital signal processing: a tutorial. IEEE Transactions on Education, 32(3), 181197.

Young, N. (1984). Linear fractional transformations in rings and modules. Linear Algebra and its Applications, $56,251-290$. 